\title{
Erratum: Correction for Incorrect Citation of Reference
}

At the reference section of the paper titled "Physiological Activities of Opuntia monacantha Haw. Fruit and Stem" published in volume 50, number 5, page 462, a reference was incorrect. It was printed as "Kim JK. "JEGUK" Opuntia ficus-indica which is new variety of Jeju Opuntia ficus-india. KR Patent 10-2061227". It should be revised as follows: "Kim JK. JEKUK Opuntia monacantha Haw. which is new variety of Jeju Baeknyuncho. KR Patent 10-2061227”.

The authors apologize to the readers for the error. 